\title{
Adipose Tissue-Derived Stem Cells from Type 2 Diabetics Reveal Conservative Alterations in Multidimensional Characteristics
}

\author{
Le Wang ${ }^{1,2,3, *}$, Leisheng Zhang ${ }^{4, *}$, Xue Liang ${ }^{2}$, Jiaqi Zou, ${ }^{1,2}$ Na Liu', Tengli Liu ${ }^{2}$, Guanqiao Wang ${ }^{2}$, \\ Xuejie Ding ${ }^{1,2}$, Yaojuan Liu ${ }^{1,3}$, Boya Zhang ${ }^{1,3}$, Rui Liang ${ }^{1,2}$, Shusen Wang,

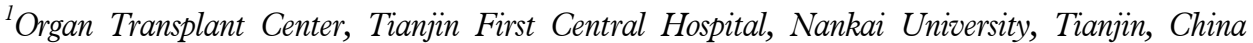 \\ ${ }^{2}$ NHC Key Laboratory for Critical Care Medicine, Tianjin, China \\ ${ }^{3}$ Tianjin Clinical Research Center for Organ Transplantation, Tianjin, China \\ ${ }^{4}$ The Postdoctoral Research Station, School of Medicine, Nankai University, Tianjin, China
}

Background and Objectives: Adipose tissue-derived mesenchymal stem cells (ASCs) are recognized as an advantaged source for the prevention and treatment of diverse diseases including type 2 diabetes mellitus (T2DM). However, alterations in characteristics of ASCs from the aforementioned T2DM patients are still obscure, which also hinder the rigorous and systematic illumination of progression and pathogenesis.

Methods and Results: In this study, we originally isolated peripancreatic adipose tissue-derived mesenchymal stem cells from both human type 2 diabetic and non-diabetic donors (T2DM-ASCs, ND-ASCs) with the parental consent, respectively. We noticed that T2DM-ASCs exhibited indistinguishable immunophenotype, cell vitality, chondrogenic differentiation and stemness as ND-ASCs. Simultaneously, there's merely alterations in migration and immunoregulatory capacities in T2DM-ASCs. However, differing from ND-ASCs, T2DM-ASCs exhibited deficiency in adipogenic and osteogenic differentiation, and in particular, the delayed cell cycle and different cytokine expression spectrum. Conclusions: The conservative alterations of T2DM-ASCs in multifaceted characteristics indicated the possibility of autologous application of ASCs for cell-based T2DM treatment in the future.

Keywords: Adipose tissue-derived mesenchymal stem cells, Type 2 diabetes mellitus, Cell vitality, Immunoregulation

Received: February 11, 2020, Revised: May 14, 2020,

Accepted: May 23, 2020, Published online: June 30, 2020

Correspondence to Rui Liang

Organ Transplant Center, Tianjin First Central Hospital, Fukang

Road 24\#, Nankai Area, Tianjin 300192, China

Tel: +86-022-23728992, Fax: +86-022-23626199

E-mail: liangrui@nankai.edu.cn

Co-Correspondence to Shusen Wang

Organ Transplant Center, Tianjin First Central Hospital, Fukang

Road 24\#, Nankai Area, Tianjin 300192, China

Tel: +86-022-23626055, Fax: +86-022-23626199

E-mail:shusen@vip.163.com

${ }^{*}$ These authors contributed equally to this work.

(a) This is an open-access article distributed under the terms of the Creative Commons Attribution Non-Commercial License (http://creativecommons.org/ licenses/by-nc/4.0/), which permits unrestricted non-commercial use, distribution, and reproduction in any medium, provided the original work is properly cited.

Copyright (C) 2020 by the Korean Society for Stem Cell Research

\section{Introduction}

Diabetes mellitus (DM), predominantly consisted of the type 1 (T1DM) and type 2 (T2DM) pathogenetic categories together with other classifications such as gestational DM and drug-induced diabetes, is acknowledged as a metabolic disease and public health concern characterized by hyperglycaemia and defects in insulin secretion and action worldwide (1-3). Of them, patients with T2DM are more pervasive (85 95\% of all cases) due to the combination of inadequate compensatory insulin secretory responses and insulin resistance at cellular level (4-6). Even though the routine therapeutic strategies, including oral antidiabetic agents along with weight management and mod- 
erate exercises, together with transplantation of insulin-producing cells (IPCs), could help partially ameliorate hyperglycaemia, yet these therapeutic regiments are largely insufficient for T2DM patients with extensive $\beta$ cell destruction and subsequent insulin deficiency $(1,3$, $7,8)$.

Mesenchymal stem cells (MSCs) are acknowledged as an advanced source with unique immunosuppressive and hematopoietic-supporting characteristics for regenerative medicine (8-12). In the 1960s, Friedenstein and his colleagues firstly reported the isolation and identification of the spindle-like MSCs from bone marrow (BM-MSCs) (13). Thereafter, MSCs have been successfully identified from various clinical specimens including adipose tissue (ASCs), placenta (P-MSCs), dental pulp (DPSCs) and umbilical cord (UC-MSCs), and even derived from human pluripotent stem cells (hPSC-MSCs) (9, 14-16). Of the above-mentioned MSCs, BM-MSCs with the most extensive applications in clinical trials whereas UC-MSCs possess the most advantageous long-term proliferation feature $(10,17)$. According to the ClinicalTrials.gov website of National Institutes of Health $(\mathrm{NIH})$, a total number of 983 registered clinical trials with MSCs have been conducted worldwide on multisystem disordering such as osteoarthritis, Crohn's disease, aplastic anaemia, cerebral stroke, diabetes mellitus, critical limb ischemia (CLI), graft-versus-host disease (GVHD) and acute-on-chronic liver failure (ACLF) $(5,7,10,11,14,18)$.

State-of-the-art updates on basic research and clinical medicine have indicated the dysfunction and correlation of MSCs in progression and pathogenesis of numerous diseases $(3,12,19,20)$. For instance, He et al. (21) verified that immune thrombocytopenia (ITP)-MSCs exhibited multifarious defects in signatures such as impaired cell vitality, increased proinflammatory factor secretion, which would accelerate disease progression of ITP patients by altering the hematopoietic microenvironment. Similar phenomenon was also observed in ASCs derived from subcutaneous adipose tissue compared with ND-ASCs $(3,22)$. With regard to DM, MSCs have been recognized as a pivotal microenvironmental ingredient for the secretion of multiple cytokines and inflammatory factors (e.g., $V E G F$, $I L-6, T N F-\alpha)$ and the transdifferentiation into insulin-secreting $\beta$ cells in the pancreas as well $(7,17,23)$. Studies on experimental type 1 diabetic mouse model showed that T1DM mice-derived BM-MSCs exhibited indistinguishable characteristics including cytomorphology, immunosuppressive and migration capabilities as the control mice but with abnormally elevated expression of mediators such as MIP-1 $\alpha / \beta, M C P-1 / 5, C C L-2, C C L-3$ and
IL-6, which facilitated recruitment and accumulation of inflammatory M1 macrophages and aggravated the occurrence of diabetic complications $(2,6,17,22)$. Yet, a considerable variety of defects were observed in T2DM-MSCs including alterations in cell proliferation, cell cycle and apoptosis, migration and multipotential differentiation, based on T2DM-derived BM-MSCs or subcutaneous adipose tissue-derived ASCs (subcutaneous ASCs) (3, 24-26). However, the current studies on T2DM-associated MSCs are still piecemeal and even controversial to some extent, and in particular, the systematically exploration of the characteristics and alterations is woefully inadequate as well $(3,20)$. Hence, there's a necessity and urgency of performing multidimensional comparation of MSCs from T2DM and non-diabetic donors (ND).

In this study, we reported the isolation and identification of type 2 diabetic adipose tissue -derived ASCs (T2DM-ASCs) and non-diabetic adipose tissue-derived ASCs (ND-ASCs). Differing from the ND-ASCs, T2DMASCs exhibited numerous defects in characteristics including the delayed cell cycle and multi-lineage differentiation. Dramatically, the T2DM-ASCs exhibited indiscriminate signatures in cytomorphology, immunophenotype, migration, chondrogenic differentiation potential together with comparable immunosuppressive property and stemness as well. Taken together, we originally isolated and systematically evaluated the phenotype of T2DM-ASCs, which also held the potential as an alternative source of MSCs for autologous application in regenerative medicine.

\section{Materials and Methods}

\section{Subjects and ethics statement}

Human peripancreatic adipose tissues were obtained from T2DM $(n=3)$ and non-diabetic organ donors $(n=5)$ with informed consents, between May 2017 and October 2019. ND-ASCs and T2DM-ASCs were extracted from the indicated adipose tissues according to the guideline of Declaration of Helsinki. All the experimental procedures were approved by the Ethics Committee of Tianjin First Central Hospital of Nankai University (ethics number: 2017N080KY). The information of the ND and T2DM donors is available in Table 1.

\section{Isolation of T2DM-ASCs and ND-ASCs}

As mentioned above, ND-ASCs and T2DM-ASCs were isolated from peripancreatic adipose tissues by enzymatic digestion. Briefly, $20 \mathrm{ml}$ adipose tissues were harvested from the candidates and washed with $1 \times$ PBS in a $50 \mathrm{ml}$ Falcon tube (Sigma-Aldrich), and digested with an equal 
Table 1. Donor information

\begin{tabular}{lcccc}
\hline & Age $(\mathrm{y})$ & $\mathrm{BMI}\left(\mathrm{kg} / \mathrm{m}^{2}\right)$ & $\mathrm{HbA} 1 \mathrm{c}(\%)$ & $\mathrm{N}$ (Male/Female) \\
\hline $\mathrm{ND}$ & $43.60 \pm 4.15$ & $24.9 \pm 1.29$ & $5.10 \pm 0.15$ & $5(3 / 2)$ \\
T2DM & $50.33 \pm 1.33$ & $27.17 \pm 0.48$ & $7.03 \pm 0.66$ & $3(2 / 1)$ \\
$\mathrm{p}$ & 0.276 & 0.244 & 0.047 & \\
\hline
\end{tabular}

Data are shown as mean \pm SEM.

volume of $1 \mathrm{mg} / \mathrm{ml}$ collagenase type I at $37^{\circ} \mathrm{C}$ for $30 \mathrm{~min}$. Then, the stromal vascular fraction cells were isolated by centrifugation at $300 \times \mathrm{g}$ at room temperature and cultured in $25 \mathrm{~cm}^{2}$ flasks in Serum-Free Human MSC Medium (Yocon) supplemented with $100 \mathrm{U} / \mathrm{ml}$ penicillin and streptomycin (Gibco). The ND-ASCs and T2DMASCs were enriched and purified by continuous passages in the abovementioned culture medium at $37^{\circ} \mathrm{C}, 5 \% \mathrm{CO}_{2}$. T2DM-ASCs and ND-ASCs at passage 2 6 were used for phenotypic analysis.

\section{Flow cytometry assay}

Flow cytometry assay were conducted as we recently described $(27,28)$. Briefly, ND-ASCs and T2DM-ASCs were dissociated into single cells by $0.25 \%$ Trypsin-EDTA (Gibco) and labelled with the indicated antibodies against CD34-APC, CD44-APC, CD45-FITC, CD73-FITC, CD90FITC, CD105-APC, together with isotype matched IgG1FITC and IgG2b-APC (BD Pharmigen), in $0.2 \%$ BSA for $30 \mathrm{~min}$ in dark and washing with $1 \times$ PBS for twice. Finally, the ND-ASCs and T2DM-ASCs were analysed by Accuri C6 Plus (BD, San Jose, CA, USA). The flow cytometry assay results were analysed with FlowJo 7.0 software (BD, San Jose, CA, USA).

\section{Cell proliferation assay}

Cell proliferation of ND-ASCs and T2DM-ASCs were conducted by utilizing the CCK-8 kit (Sigma-Aldrich, St. Louis, USA) according to the manufacturer's instructions. In brief, $5 \times 10^{3}$ ND-ASCs and T2DM-ASCs were seeded in 96-well plates $(100 \mu \mathrm{l} /$ well $)$ at a density of per well with 5 replications for each time point $(4 \mathrm{~h}, 24 \mathrm{~h}, 48 \mathrm{~h}$, $72 \mathrm{~h}$ ) at $37^{\circ} \mathrm{C}, 5 \% \mathrm{CO}_{2}$. After that, ND-ASCs and T2DMASCs were incubated with $10 \mu 1$ CCK- 8 solution at $37^{\circ} \mathrm{C}$ for an additional $2 \mathrm{~h}$. The absorbance of the solutions was measured at $450 \mathrm{~nm}$ using a microplate absorbance reader (Bio-Rad, California, USA).

\section{Cell apoptosis assay}

ND-ASCs and T2DM-ASCs were collected and incubated with Annexin V and 7-AAD (BD) for $15 \mathrm{~min}$ at
RT according to the $\mathrm{BD}$ manufacture's instruction. The proportion of apoptotic ND-ASCs and T2DM-ASCs were quantified by flow cytometry analysis using an Accuri C6 plus instrument (BD, San Jose, CA, USA). All flow cytometry assay data were analysed with FlowJo 7.0 software (BD, San Jose, CA, USA).

\section{Cell cycle assay}

ND-ASCs and T2DM-ASCs were collected and fixed in $70 \%$ ethanol for at least $2 \mathrm{~h}$ at $4{ }^{\circ} \mathrm{C}$. After washing with $1 \times$ PBS for twice, ND-ASCs and T2DM-ASCs were incubated for $30 \mathrm{~min}$ at room temperature with $10 \mu \mathrm{g} / \mathrm{ml}$ propidium iodide (PI, Beyotime) staining solution. The distribution of multiple cell cycle phases was quantified by flow cytometry using an Accuri C6 plus instrument (BD, San Jose, CA, USA).

\section{Adipogenic differentiation}

Adipogenic differentiation was conducted as we recently reported with several modification (10). In details, NDASCs and T2DM-ASCs were seeded at a density of $2 \times 10^{4}$ cells $/ \mathrm{cm}^{2}$ at $37^{\circ} \mathrm{C}, 5 \% \mathrm{CO}_{2}$. When cells reached $80 \%$ confluent, the supernatant was discarded and changed into adipogenic differentiation medium containing $1 \mu \mathrm{M}$ dexamethasone, $0.1 \mathrm{mM}$ indomethacin, $0.5 \mathrm{mM}$ 3-isobutyl1-methylxanthine, and $10 \mathrm{mg} / \mathrm{L}$ insulin in Serum-free human MSC medium (Yocon). The medium was subsequently changed twice a week. After 21 days, adipogenic differentiation was assessed by Oil Red $\mathrm{O}$ staining of the fat droplets. The phase contrast image was photographed with Nikon ElipseTi-U microscope (Nikon, Tokyo, Japan).

\section{Osteogenic differentiation}

Osteogenic differentiation was conducted as we previously described with several modification (10). In details, ND-ASCs and T2DM-ASCs were seeded at a density of $2 \times 10^{4}$ cells $/ \mathrm{cm}^{2}$ at $37^{\circ} \mathrm{C}, 5 \% \mathrm{CO}_{2}$. When cells reached $80 \%$ confluent, the supernatant was discarded and changed into osteogenic differentiation medium containing 100 $\mathrm{nM}$ dexamethasone, $50 \mu \mathrm{M}$ L-ascorbic acid 2-phosphate, and $10 \mathrm{mM} \beta$-glycerophosphate in Serum-free human MSC medium (Yocon). The medium was subsequently changed twice a week. After 28 days, osteogenic differentiation was assessed by von Kossa staining (Abcam). The phase contrast image was photographed with Nikon ElipseTi-U microscope (Nikon, Tokyo, Japan).

\section{Chondrogenic differentiation}

Chondrogenic differentiation was performed as we recently reported (10). Briefly, ND-ASCs and T2DM-ASCs 
were seeded at a density of $2 \times 10^{4}$ cells $/ \mathrm{cm}^{2}$ at $37^{\circ} \mathrm{C}, 5 \%$ $\mathrm{CO}_{2}$. When cells reached $80 \%$ confluent, the supernatant was discarded and changed into chondrogenic differentiation medium (StemCells). The medium was subsequently changed twice a week. After 28 days, chondrogenic differentiation was assessed by Alcian Blue staining (Abcam). The phase contrast image was photographed with Nikon ElipseTi-U microscope (Nikon, Tokyo, Japan).

\section{Quantitative real-time PCR (qRT-PCR) assay}

Total RNA extraction and cDNA synthesis were performed by utilizing the RNeasy Mini Kit (QIAGEN) and PrimeScript RT reagent Kit with gDNA Eraser (Takara) as we described (23). qRT-PCR was performed with SYBR Premix ExTaq II (Takara) and LightCycler96 System (Roche). Relative mRNA expression of the indicated genes was calculated by the $2^{-\triangle \mathrm{CT}}$ method. GAPDH was used as an internal control. The Primer sequences are listed in Table 2.

\section{Inhibition of lymphocyte proliferation by ASCs}

Inhibition of lymphocyte proliferation to human peripheral blood mononuclear cells (PBMCs) by the indicated ASCs. In brief, PBMCs were isolated from peripheral blood of healthy donors by standard Ficoll density gradient centrifugation. Then, the prepared PBMCs were cocultured with or without $5 \times 10^{4} \mathrm{ND}$-ASCs or T2DMASCs at a ratio of $5: 1$ in 96-well plates at $37^{\circ} \mathrm{C}, 5 \% \mathrm{CO}_{2}$ in the presence of $5 \mathrm{ng} / \mu \mathrm{l}$ PHA. 3 days later, PBMCs in the supernatant were collected and labelled with antibodies against CD3 and analysed by Accuri C6 Plus (BD, San Jose, CA, USA) and Flowjo software (BD, San Jose, CA, USA).

\section{Wound healing assay}

ND-ASCs or T2DM-ASCs were seeded in 6-well plate, when the ND-ASCs or T2DM-ASCs reached $80 \%$ confluent, the indicated cell layers were scratched with $200 \mu 1$ tips and captured with a Nikon ElipseTi-U microscope (Nikon, Tokyo, Japan) at the indicated time points $(0 \mathrm{~h}$, $12 \mathrm{~h}, 24 \mathrm{~h}$ ). Images of the scratch area were captured and analyzed by the Image J software.

\section{Statistical analysis}

Statistical analysis in the study was conducted by utilizing Prism 5 software (GraphPad, San Diego, USA). The data were presented as means \pm SEM. Student's t-test was used to analyse the data of the two indicated groups. $\mathrm{p}<$ 0.05 was considered statistically significant. ${ }^{*}, \mathrm{p}<0.05$; $* *, \mathrm{p}<0.01 ; * * *, \mathrm{p}<0.001 ; \mathrm{NS}$, not significant.
Table 2. Human genes primer sequences for qRT-PCR

\begin{tabular}{|c|c|}
\hline Genes & Primers $\left(5^{\prime}-3^{\prime}\right)$ \\
\hline$B G L A P-F$ & GGCGCTACCTGTATCAATGG \\
\hline$B G L A P-\mathrm{R}$ & TCAGCCAACTCGTCACAGTC \\
\hline PPAR- $\gamma-\mathrm{F}$ & GCTGGСCTCCTTGATGAATA \\
\hline$P P A R-\gamma-\mathrm{R}$ & TGTCTTCAATGGGCTTCACA \\
\hline ACAN-F & ССССТGСТАTTTCATCGACСC \\
\hline ACAN-R & GACACACGGCTCCACTTGAT \\
\hline POU5F1-F & CTTGAATCCCGAATGGAAAGGG \\
\hline POU5F1-R & GTGTATATCCCAGGGTGATCCTC \\
\hline NANOG-F & TTTGTGGGCCTGAAGAAAACT \\
\hline NANOG-R & AGGGCTGTCCTGAATAAGCAG \\
\hline IDO1-F & TGGGGCAAAGGTCATGGAG \\
\hline IDO1-R & TTTCTTGGAGAGTTGGCAGTAAG \\
\hline$S D F-1-\mathrm{F}$ & ССGСGСТСТGССТCAGCGACGGGAAG \\
\hline$S D F-1-R$ & СТTGTTTAAAGCTTTCTCCAGGTACT \\
\hline CXCR4-F & AGCTGTTGGTGAAAAGGTGGTCTATG \\
\hline CXCR4-R & GCGCTTCTGGTGGCCCTTGGAGTGTG \\
\hline IGF-1-F & AGCCTCCTGTGAAAGTGACG \\
\hline IGF-1-R & GTGCTTCCTTGTAGTAAACGG \\
\hline$T G F-\beta-F$ & AAATGGATACACCGAACCCAA \\
\hline$T G F-\beta-\mathrm{R}$ & GCTGCATTTGCAAGACTTTAC \\
\hline HGF-F & GCTATCGGGGTAAAGACCTACA \\
\hline HGF-R & CGTAGCGTACCTCTGGATTGC \\
\hline TSG-6-F & TGTCTGTGCTGCTGGATGGAT \\
\hline TSG-6-R & TGTGGGTTGTAGCAATAGGCAT \\
\hline$I L-1 R a-\mathrm{F}$ & GAAGATGTGCCTGTCCTGTGTC \\
\hline$I L-1 R \alpha-\mathrm{R}$ & СGCTTGTCCTGCTTTCTGTTC \\
\hline GAPDH-F & CATGTTCGTCATGGGTGTGAACCA \\
\hline GAPDH-R & ATGGCATGGACTGTGGTCATGAGT \\
\hline
\end{tabular}

\section{Results}

\section{T2DM-ASCs show similarities with ND-ASCs in cytomorphology and immunophenotype}

To clarify the underlying type 2 diabetes-associated pathogenesis and uncover the potential defects of MSCs during disease progression, we originally isolated and cultured the peripancreatic adipose tissue-derived MSCs from 3 T2DM (T2DM-ASCs) and 5 non-diabetic donors (ND-ASCs), according to the Declaration of Helsinki and the Ethics Committee of Tianjin First Central Hospital (Table 1). Compared with ND-ASCs, T2DM-ASCs exhibited indistinguishable spindle-like cytomorphology after in vitro passages for 3 times (Fig. 1A). Flow cytometry analysis showed that both T2DM-ASCs and ND-ASCs expressed high level of MSC-associated biomarkers (CD44, CD73, CD90, CD105) (>95\%), whereas with minimal expression of hematopoietic or endothelial-associated biomarkers (CD34, CD45) (Fig. 1B and 1C). Taken together, we successfully obtained T2DM-ASCs and ND-ASCs from 
A

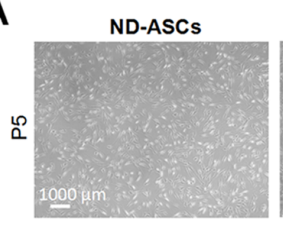

B

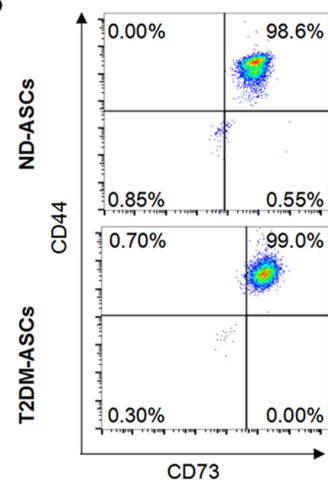

T2DM-ASCs

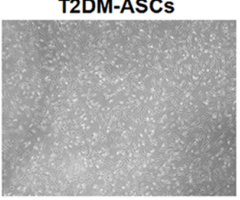

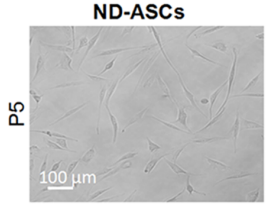
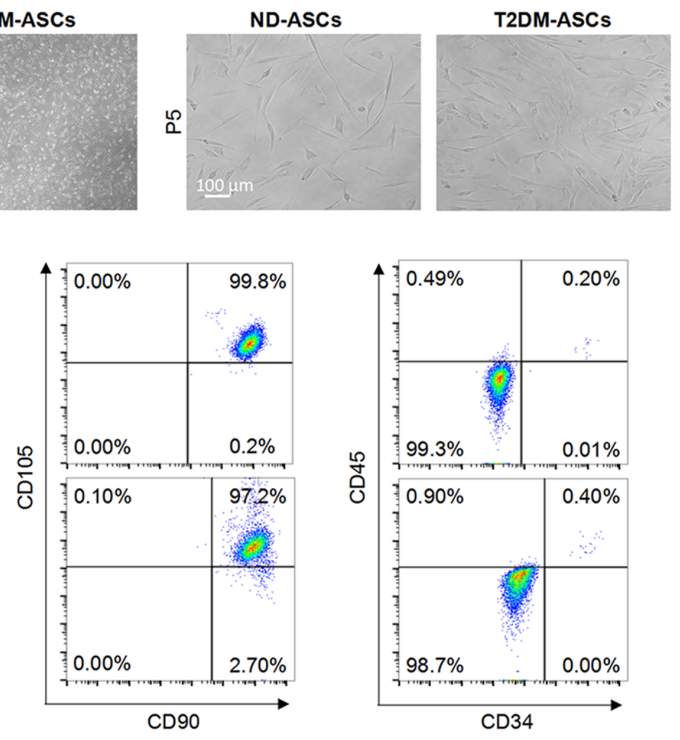

C

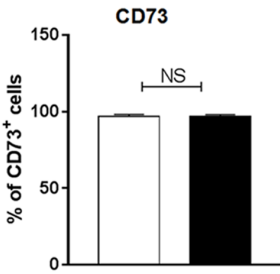

CD105

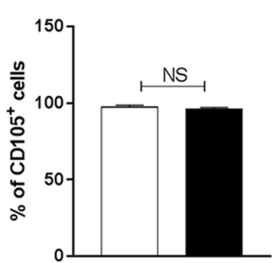

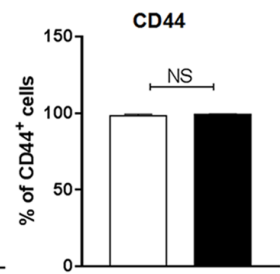

CD34

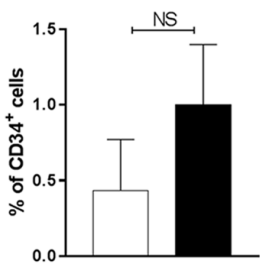

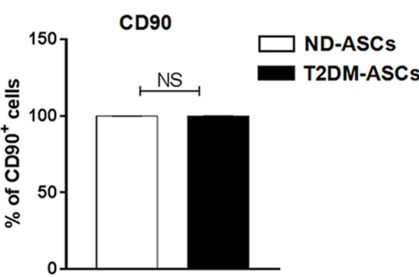

CD45

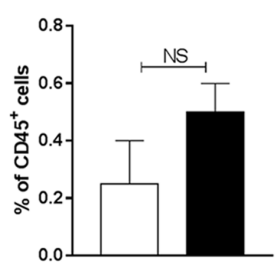

Fig. 1. T2DM-ASCs show similar cytomorphology and immunophenotype. (A) Morphology of ND-ASCs and T2DM-ASCs under same conditions (left, 40× magnification; right, $100 \times$ magnification,). (B, C) Immunophenotype of ND-ASCs $(n=5)$ and T2DM-ASCs $(n=3)$ at P6, analysed by flow cytometry. The markers including CD44, CD73, CD90, CD105, CD34 and CD45. The data were shown as the mean \pm SEM, each experiment was repeated at least twice, one representative example shown. NS: not significant. peripancreatic adipose tissue, whereas the indicated ASCs showed indistinguishable cytomorphology and immunophenotype.

\section{T2DM-ASCs and ND-ASCs exhibit indiscriminate signatures in cell viability}

We then speculated whether the cell viability of the two ASCs was distinguished from each other. For the purpose, we originally conducted cell count analysis and found that T2DM-ASCs and ND-ASCs showed comparable cell proliferation capacity, which was further verified by the growth curve (Fig. 2A and 2B). With the aid of flow cytometry analysis, we found T2DM-ASCs showed a cell cycle arrest at G0/G1 phases and a reduction in G2/M phases when compared with ND-ASCs, which indicated the impaired cell cycle in T2DM-ASCs (Fig. 2C and 2D). Unexpectedly, the proportion of population with early apoptotic (Annexin $\mathrm{V}^{+} 7-\mathrm{AAD}^{-}$) or apoptotic (Annexin
$\mathrm{V}^{+}$) property in T2DM-ASCs was consistent with that in the ND-ASCs group, which was identified by 7-AAD and Annexin $\mathrm{V}$ staining (Fig. 2E and $2 \mathrm{~F}$ ). In summary, the isolated T2DM-ASCs held similarities with ND-ASCs in most of cell viability except for the distribution of multiple cell cycle phases.

\section{ND-ASCs possess preferable adipogenic and osteogenic differentiation capacities over T2DM-ASCs in vitro}

To further illuminate the potential distinctions in biological signatures, we subsequently detected the adipogenic, osteogenic and chondrogenic differentiation capacity of T2DM-ASCs and ND-ASCs. As shown by Oil Red S staining, fewer typical adipose droplets were generated from T2DM-ASCs after 21 days of adipogenic differentiation compared with the ND-ASCs group (Fig. 3A, Supplementary Fig. S1A). Quantitative analysis of adipo- 
A

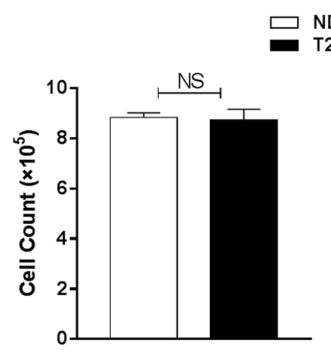

C

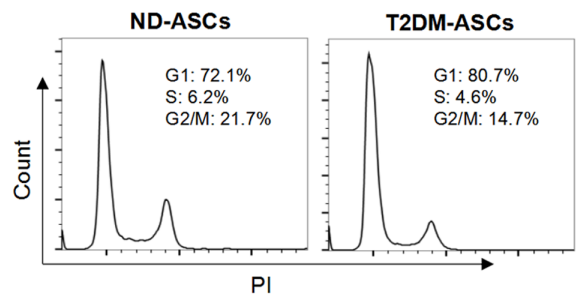

E

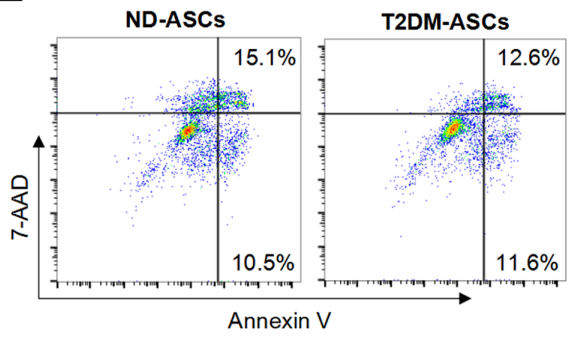

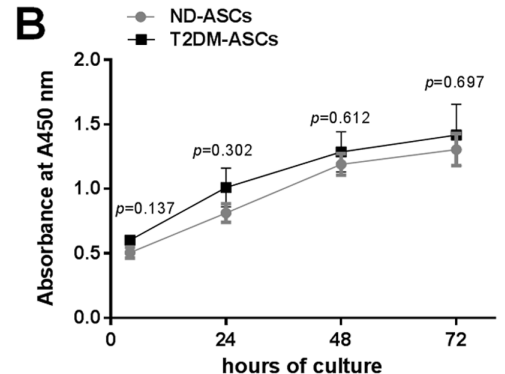

D

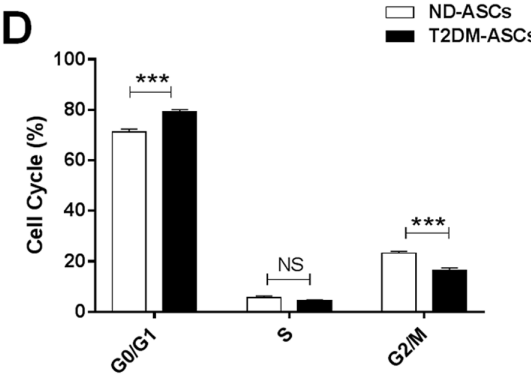

$\mathbf{F}$

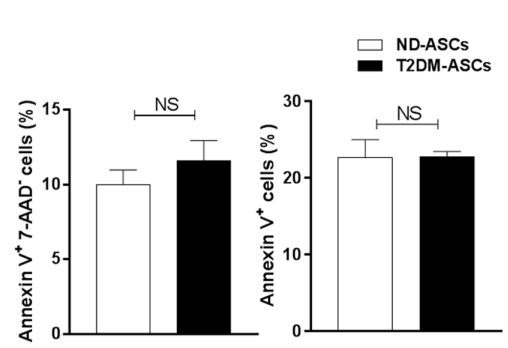

Fig. 2. T2DM-ASCs exhibit indiscriminate signatures in cell viability. (A) Cell count of ND-ASCs $(n=4)$ and T2DM-ASCs $(n=3)$ at P5. (B) CCK-8 assay of ND-ASCs $(n=3)$ and T2DM-ASCs for 4, 24, 48 and $72 \mathrm{~h}$. (C) PI staining profiles of ND-ASCs $(n=4)$ and T2DM-ASCs $(n=3)$ by flow cytometry. (D) Histograms statistics of cell cycle status populations. (E) 7-AAD and Annexin $V$ staining profiles of ND-ASCs $(n=4)$ and T2DM-ASCs $(n=3)$ by flow cytometry. (F) Histograms statistics of cell apoptosis, including early apoptosis (Annexin $\mathrm{V}^{+} 7-\mathrm{AAD}^{-}$) and total apoptosis (Annexin $\mathrm{V}^{+}$) populations. The data were shown as the mean \pm SEM, each experiment was repeated at least twice, one representative example shown. NS: not significant. $* * * \mathrm{p}<0.001$. genic markers (e.g., $P P A R-\gamma$ ) further confirmed the decline of adipocyte generation in the T2DM-ASCs group (Fig. 3B). Interestingly, ND-ASCs-derived cells also showed preferable osteogenic differentiation capacity than that in the T2DM-ASCs group as indicated by von Kossa staining (Fig. 3C, Supplementary Fig. S1B) and expression of osteogenic marker, BGLAP (Fig. 3D). Differing from the aforementioned adipogenic and osteogenic differentiation, T2DM-ASCs revealed indistinguishable chondrogenic differentiation potential as confirmed by Alcian Blue staining (Fig. 3E, Supplementary Fig. S1C) and ACAN mRNA expression (Fig. 3F). Hence, T2DM-ASCs possessed impaired adipogenic and osteogenic differentiation potential to the ND-ASCs group.

\section{T2DM-ASCs show comparable migration and immunosuppressive capacity as ND-ASCs}

To date, we and other investigators have manifested the alterations of ASCs during multiple pathological processes including cell migration, immunosuppressive capacity, together with numerous cytokine secretion. Thus, we primitively took advantage of wound healing analysis and found that T2DM-ASCs with attenuated migration capacity com- pared with that in ND-ASCs (Fig. 4A and 4B). Simultaneously, by conducting the coculture of ASCs with peripheral blood mononuclear cells (PBMCs), we noticed that the inhibitory capacity of T2DM-ASCs on proliferation of $\mathrm{CD}^{+} \mathrm{T}$ cells was comparable to the PBMCs $+\mathrm{PHA}+\mathrm{ND}-$ ASCs group (Fig. 4C and 4D). Collectively, T2DM-ASCs exhibited indiscriminate signatures with ND-ASCs in migration and immunoregulation.

\section{T2DM-ASCs reveal multifaceted characteristics in cytokine expression with ND-ASCs but conserved stemness}

Having primitively verified the indiscriminate immunosuppression upon PBMC proliferation, we next are wondering about the expression levels of cytokines as well as inflammatory factors between the two indicated ASCs. For the purpose, we conducted quantitative analysis of a variety of cytokines in ND-ASCs and T2DM-ASCs, and found there were no discernible differences in IDOI, SDF-1 or CXCR4 expression (Fig. 5A). Meanwhile, besides those with no statistically significant differences in expression, we also noticed the alterations such as $H G F$, $T G F-\beta, I G F-1, I L-1 R a$ and TSG-6, which were involved 
A

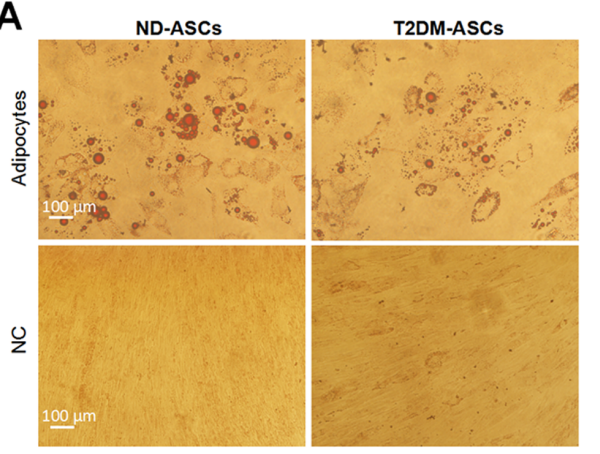

C

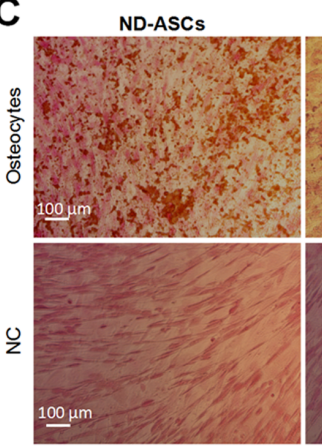

E

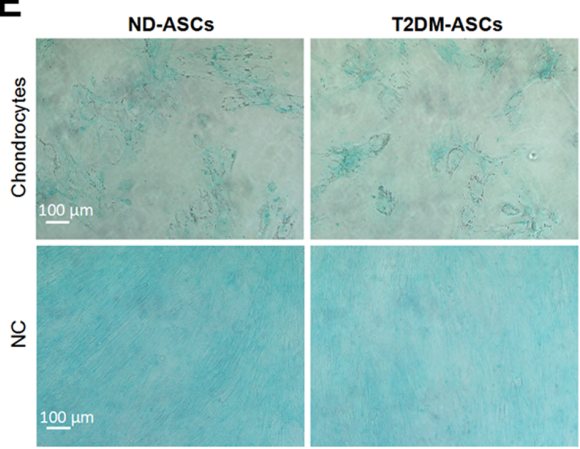

B

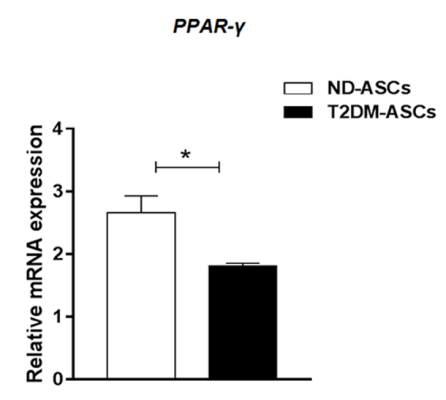

D

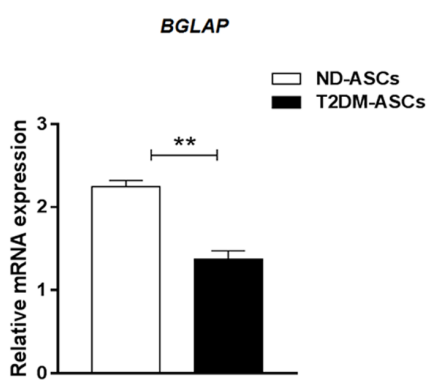

$\mathbf{F}$

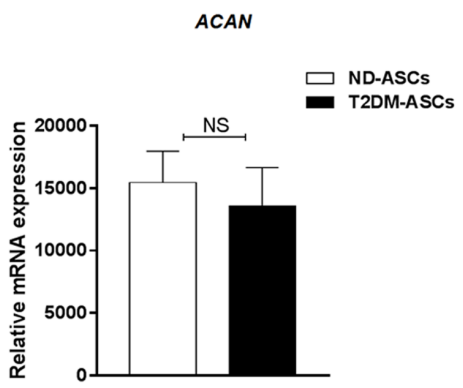

Fig. 3. T2DM-ASCs possess impaired adipogenic and osteogenic differentiation potential. (A) The morphology of adipogenic differentiation of ND-ASCs $(n=3)$ and T2DM-ASCs $(\mathrm{n}=3)$ by Oil Red O staining $(200 \times$ magnification). (B) qRT-PCR assessment of adipogenic marker PPAR- $\gamma$. (C) The morphology of osteogenic differentiation of ND-ASCs $(n=3)$ and T2DM-ASCs $(n=3)$ by von Kossa staining (200× magnification). (D) qRT-PCR assessment of osteogenic marker BGLAP. (E) The morphology of chondrogenic differentiation of ND-ASCs $(\mathrm{n}=3)$ and T2DM-ASCs $(n=3)$ by Alcian Blue staining $(200 \times$ magnification). (F) qRT-PCR assessment of chondrogenic marker ACAN. All values are normalized to the negative control (NC) group. The data were shown as the mean \pm SEM, each experiment was repeated at least twice, one representative example shown. ${ }^{*} \mathrm{p}<0.05,{ }^{* *} \mathrm{p}<0.01$. in regulation of islet cell function (Fig. 5B). However, the expression levels of stemness-associated biomarkers (POU5F1, NANOG) in ND-ASCs and T2DM-ASCs at various passages (P2, P6) didn't show statistically significant difference in vitro, which confirmed the retained property of T2DM-ASCs in the stemness potential (Fig. 5C).

\section{Discussion}

Longitudinal studies have demonstrated adipose tissue as an easily accessible source of high-quality mesenchymal stem cells (MSCs) with extensive prospects for clinical applications, which are also acknowledged as a pivotal microenvironmental component involved in numerous disease progression $(5,11,19,21)$. However, there's merely reports on stem cell identification from T2DM- or ND-derived peripancreatic adipose tissues (2). Above all, the sys- tematic comparation of T2DM-ASCs with ND-ASCs is largely unprocurable as well. Therefore, we originally isolated the indicated peripancreatic ASCs and comprehensively investigated the signatures to dissect the alterations occurred in T2DM-ASCs. For instance, compared with ND-ASCs, these T2DM-ASCs exhibited impaired adipogenic and osteogenic differentiation properties, together with delayed cell cycle. Hence, our findings have provided new evidence for the defects of T2DM-ASCs, together with the explanation to pathogenesis of type 2 diabetes mellitus as well.

For long stretches, to our knowledge, no reports have been documented on isolating T2DM-ASCs from peripancreatic adipose tissue $(4,7)$. For decades, we and other investigators have identified ASCs from subcutaneous adipose tissue for applications in regenerative medicine $(3,5)$. However, in this study, the identified T2DM-ASCs were 
A

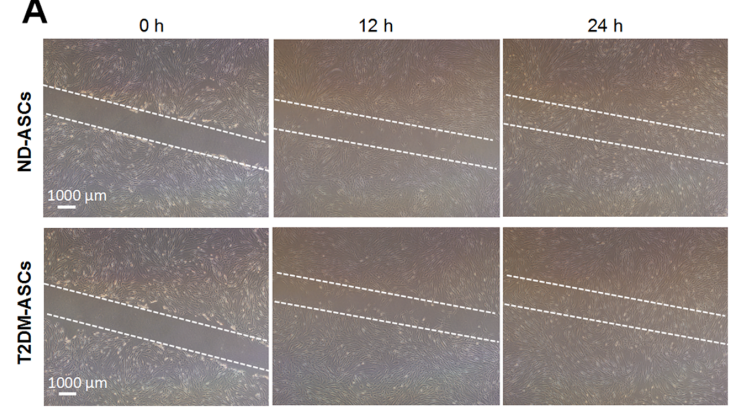

C

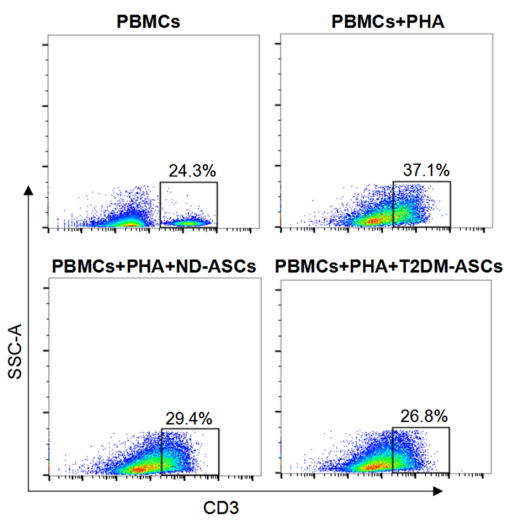

A

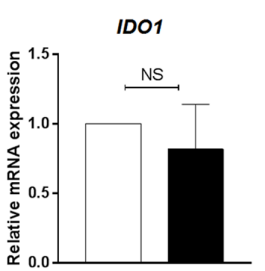

B
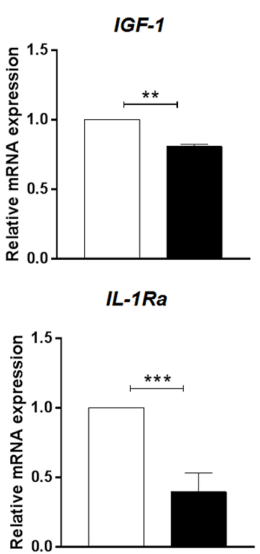

C

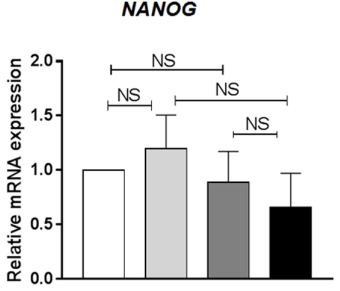

B

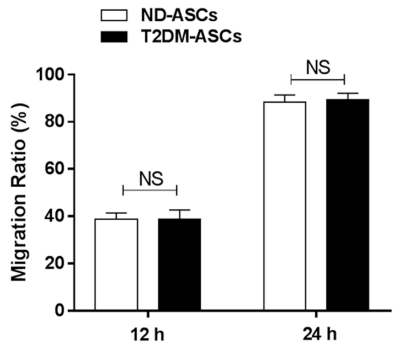

D

$\square$ Unstimulated PBMCs

- PBMCs+PHA

$\sqsupseteq$ PBMCs+PHA+ND-ASCs

In PBMCs+PHA+T2DM-ASC

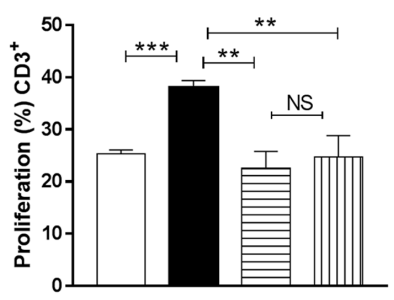

Fig. 4. T2DM-ASCs show comparable migration and immunosuppressive capacity. (A) Cell migration ability of ND-ASCs $(n=3)$ and T2DMASCs $(n=3)$ at 0,12 and 24 hours (40×magnification). (B) Statistical analysis of cell migration area by the ImageJ. (C) ND-ASCs $(\mathrm{n}=3)$ and T2DM-ASCs $(n=3)$ cocultured with PBMCs for 3 days, flow cytometry analysis of PBMCs with CD3 staining. (D) Percentages of $\mathrm{CD}^{+}$lymphocyte proliferation. The data were shown as the mean \pm SEM, each experiment was repeated at least twice, one representative example shown. NS: not significant. ${ }^{* *} \mathrm{p}<0.01,{ }^{* * *} \mathrm{p}<0.001$.

Fig. 5. The characteristics of cytokine expression and stemness of T2DM-ASCs and ND-ASCs. (A, B) The comparation of immunomodulation-associated factors (A) and islet function-related factors (B) between T2DM-ASCs $(\mathrm{n}=3)$ and ND-ASCs $(n=3)$. (C) The comparation of stemness-associated gene expression (NANOG, POU5F1) between T2DMASCs $(n=3)$ and ND-ASCs $(n=3)$. The data were shown as the mean \pm SEM, each experiment was repeated at least twice. NS: not significant. ${ }^{* *} \mathrm{p}<0.01,{ }^{* * *} \mathrm{p}<0.001$. 
isolated from the peripancreatic adipose tissue of type 2 diabetesmellitus, which was distinguished from the reported ASCs. For instance, the subcutaneous adipose tissue-derived ASCs from T2DM patients possessed enhanced differentiation capacity into adipocytes whereas declined potential into osteoblasts (24). The contrarious conclusions might be largely ascribed to the sophisticated and polytropic microenvironment induced by inflammatory factors or metabolites during the disease progression $(11,20,25)$. For example, diabetes and obesity could contribute to local hypoxia in adipose tissue and increase the secretion of hormones, growth factors, and adipokines to initiate the proliferation, adipogenic differentiation or microvessel formation of ASCs, together with the immune properties (10, 29-31). Although it has been reported that a short term of high glucose stimulation reduced proliferation of gestational-MSCs but enhanced adipogenic differentiation in vitro (32), our results demonstrated that the peripanceatic diabetic milieus, including hyperglycemia, cause cell cycle delay as well as compromised adipogenic and osteogenic differentiation of T2DM-ASCs in the long run. Overall, there's no surprises that ASCs with different origins might exhibit dissimilar biological potentials, which has been demonstrated among subtypes of MSCs such as the above-mentioned UC-MSCs, P-MSCs and BM-MSCs $(8,10,17)$. In addition, the defects in cell vitality including the delayed cell cycle between T2DMASCs and ND-ASCs in this study were consist with current reports that hypoxia and hyperglycaemia didn't affect proliferation of ASCs but "stemness" (12, 20, 26, 33).

MSCs have been acknowledged as a splendid candidate source for disease managements such as hematologic malignancies, graft-versus-host disease (GVHD), acute myocardial infarction, liver cirrhosis and diabetes mellitus (4, $5,11,18,27)$. To date, ASCs together with other tissue derived MSCs, including the non-invasive umbilical cord-derive MSCs (UC-MSCs), placental-derived MSCs (P-MSCs) and dental pulp stem cells (DPSCs) together with even pluripotent stem cell-derived MSCs (PSCMSCs), have been demonstrated preferable alternatives of the invasive bone marrow-derived MSCs (BM-MSCs) for future clinical applications $(5,9,11)$. However, systematic and detailed dissection of T2DM-ASCs both at the cellular and molecular levels is fundamental and prerequisite to discover the proofs on pathogenesis of diabetes mellitus (7, 19). For instance, Wang et al. (23) and Krishnan et al. (34) reported that intravenous injection of ASCs was sufficient to improve insulin sensitivity and alleviate $\beta$ cell death and even ameliorate hippocampal dysfunction of T2DM percipient mice, respectively. Congruously, we currently verified that MSCs participated in the restorative process of dysfunctional type 2 diabetic islets by reversing $\beta$ cell dedifferentiation, yet the underling pathogenesis of T2DM-ASCs involved in the progression and efficacy still need further systematic and in-depth research (23). Here, the present study further dissected the potential of T2DM-ASCs in the treatment of T2DM. MSCs ameliorate islet dysfunction mainly through its paracrine function, secreting factors modulating inflammation and immunity, as reviewed in previous studies (3, 35-37). Some of these MSCs-secreted factors have been reported improving $\beta$ cell function, proliferation or viability, such as IGF (38), HGF (39) and IL-1Ra (23). Thus we evaluated the gene expression of these factors and results showed that $I G F-1, H G F$, and IL-IRa were significantly downregulated in T2DM-ASCs, while the factors that modulating immunity have no significant change, suggesting a possibility of compromised ability of T2DM-ASCs in improving the islet pathology of T2DM patients.

In the present study, we have isolated and identified human T2DM-ASCs and ND-ASCs, respectively. Compared with ND-ASCs, T2DM-ASCs show limited multifaceted alterations in biological signatures including multi-differentiation potentials, cell vitality, PBMC inhibition and stemness together with indistinguishable characteristics as ND-ASCs as well. Above all, the minimal and conservative alterations of T2DM-ASCs in multifaceted characteristics also indicated the possibility of autologous application of ASCs for cell-based T2DM treatment in the future, yet there is still a need to develop strategies to further improve T2DM-ASCs functions.

\section{Acknowledgments}

The work was supported by the National Natural Science Foundation of China (81870535), Key projects of Tianjin Natural Science Foundation (18JCZDJC33100), Chun Foundation of Tianjin First Central Hospital (2019CM01 and 2019CF28), Annual scientific and technological activities for overseas graduates in Tianjin (2019), China Postdoctoral Science Foundation (2019M661033), Natural Science Foundation of Tianjin (19JCQNJC12500).

\section{Potential Conflict of Interest}

The authors have no conflicting financial interest.

\section{Supplementary Materials}

Supplementary data including one figure can be found with this article online at http://pdf.medrang.co.kr/paper/ 
pdf/IJSC/IJSC-13-s20028.pdf.

\section{References}

1. Yang W, Lu J, Weng J, Jia W, Ji L, Xiao J, Shan Z, Liu J, Tian H, Ji Q, Zhu D, Ge J, Lin L, Chen L, Guo X, Zhao Z, Li Q, Zhou Z, Shan G, He J; China National Diabetes and Metabolic Disorders Study Group. Prevalence of diabetes among men and women in China. $\mathrm{N}$ Engl J Med 2010;362:1090-1101

2. He X, Yang Y, Yao MW, Ren TT, Guo W, Li L, Xu X. Full title: High glucose protects mesenchymal stem cells from metformin-induced apoptosis through the AMPKmediated mTOR pathway. Sci Rep 2019;9:17764

3. Zang L, Hao H, Liu J, Li Y, Han W, Mu Y. Mesenchymal stem cell therapy in type 2 diabetes mellitus. Diabetol Metab Syndr 2017;9:36

4. Cho J, D'Antuono M, Glicksman M, Wang J, Jonklaas J. A review of clinical trials: mesenchymal stem cell transplant therapy in type 1 and type 2 diabetes mellitus. Am J Stem Cells 2018;7:82-93

5. Qi Y, Ma J, Li S, Liu W. Applicability of adipose-derived mesenchymal stem cells in treatment of patients with type 2 diabetes. Stem Cell Res Ther 2019;10:274

6. Ying W, Fu W, Lee YS, Olefsky JM. The role of macrophages in obesity-associated islet inflammation and $\beta$-cell abnormalities. Nat Rev Endocrinol 2020;16:81-90

7. Holmes D. Diabetes: MSC transplant prevents $\beta$-cell dysfunction. Nat Rev Endocrinol 2014;10:701

8. Samsonraj RM, Raghunath M, Nurcombe V, Hui JH, van Wijnen AJ, Cool SM. Concise review: multifaceted characterization of human mesenchymal stem cells for use in regenerative medicine. Stem Cells Transl Med 2017;6:21732185

9. Zhang L, Wang H, Liu C, Wu Q, Su P, Wu D, Guo J, Zhou W, Xu Y, Shi L, Zhou J. MSX2 Initiates and accelerates mesenchymal stem/stromal cell specification of hPSCs by regulating TWIST1 and PRAME. Stem Cell Reports 2018; 11:497-513

10. Zhao Q, Zhang L, Wei Y, Yu H, Zou L, Huo J, Yang H, Song B, Wei T, Wu D, Zhang W, Zhang L, Liu D, Li Z, Chi Y, Han Z, Han Z. Systematic comparison of hUCMSCs at various passages reveals the variations of signatures and therapeutic effect on acute graft-versus-host disease. Stem Cell Res Ther 2019;10:354

11. Kabat M, Bobkov I, Kumar S, Grumet $M$. Trends in mesenchymal stem cell clinical trials 2004-2018: is efficacy optimal in a narrow dose range? Stem Cells Transl Med 2020;9: $17-27$

12. Shi Y, Wang Y, Li Q, Liu K, Hou J, Shao C, Wang Y. Immunoregulatory mechanisms of mesenchymal stem and stromal cells in inflammatory diseases. Nat Rev Nephrol 2018;14:493-507

13. Friedenstein AJ, Petrakova KV, Kurolesova AI, Frolova GP. Heterotopic of bone marrow. Analysis of precursor cells for osteogenic and hematopoietic tissues. Transplantation 1968;
6:230-247

14. Wei Y, Hou H, Zhang L, Zhao N, Li C, Huo J, Liu Y, Zhang W, Li Z, Liu D, Han Z, Zhang L, Song B, Chi Y, Han Z. JNKi- and DAC-programmed mesenchymal stem/ stromal cells from hESCs facilitate hematopoiesis and alleviate hind limb ischemia. Stem Cell Res Ther 2019;10:186

15. Zhang X, Yang Y, Zhang L, Lu Y, Zhang Q, Fan D, Zhang Y, Zhang Y, Ye Z, Xiong D. Mesenchymal stromal cells as vehicles of tetravalent bispecific Tandab (CD3/CD19) for the treatment of $\mathrm{B}$ cell lymphoma combined with IDO pathway inhibitor D-1-methyl-tryptophan. J Hematol Oncol 2017;10:56

16. Yao J, Chen N, Wang X, Zhang L, Huo J, Chi Y, Li Z, Han Z. Human supernumerary teeth-derived apical papillary stem cells possess preferable characteristics and efficacy on hepatic fibrosis in mice. Stem Cells Int 2020;2020: 6489396

17. Pourgholaminejad A, Aghdami N, Baharvand H, Moazzeni SM. The effect of pro-inflammatory cytokines on immunophenotype, differentiation capacity and immunomodulatory functions of human mesenchymal stem cells. Cytokine 2016;85:51-60

18. McGonagle D, Baboolal TG, Jones E. Native joint-resident mesenchymal stem cells for cartilage repair in osteoarthritis. Nat Rev Rheumatol 2017;13:719-730

19. Kfoury Y, Scadden DT. Mesenchymal cell contributions to the stem cell niche. Cell Stem Cell 2015;16:239-253

20. Nombela-Arrieta C, Ritz J, Silberstein LE. The elusive nature and function of mesenchymal stem cells. Nat Rev Mol Cell Biol 2011;12:126-131

21. He Y, Xu LL, Feng FE, Wang QM, Zhu XL, Wang CC, Zhang JM, Fu HX, Xu LP, Liu KY, Huang XJ, Zhang XH. Mesenchymal stem cell deficiency influences megakaryocytopoiesis through the TNFAIP3/NF- $\kappa \mathrm{B} / \mathrm{SMAD}$ pathway in patients with immune thrombocytopenia. $\mathrm{Br} \mathrm{J}$ Haematol 2018;180:395-411

22. Weisberg SP, McCann D, Desai M, Rosenbaum M, Leibel RL, Ferrante AW Jr. Obesity is associated with macrophage accumulation in adipose tissue. J Clin Invest 2003;112: 1796-1808

23. Wang L, Liu T, Liang R, Wang G, Liu Y, Zou J, Liu N, Zhang B, Liu Y, Ding X, Cai X, Wang Z, Xu X, Ricordi C, Wang S, Shen Z. Mesenchymal stem cells ameliorate $\beta$ cell dysfunction of human type 2 diabetic islets by reversing $\beta$ cell dedifferentiation. EBioMedicine 2020;51:102615

24. Kočí Z, Turnovcová K, Dubský M, Baranovičová L, Holáň V, Chudíčková M, Syková E, Kubinová S. Characterization of human adipose tissue-derived stromal cells isolated from diabetic patient's distal limbs with critical ischemia. Cell Biochem Funct 2014;32:597-604

25. Barbagallo I, Li Volti G, Galvano F, Tettamanti G, Pluchinotta FR, Bergante S, Vanella L. Diabetic human adipose tissue-derived mesenchymal stem cells fail to differentiate in functional adipocytes. Exp Biol Med (Maywood) 2017;242:1079-1085

26. Alicka M, Major P, Wysocki M, Marycz K. Adipose-derived 
mesenchymal stem cells isolated from patients with type 2 diabetes show reduced "stemness" through an altered secretome profile, impaired anti-oxidative protection, and mitochondrial dynamics deterioration. J Clin Med 2019;8:765

27. Lim M, Wang W, Liang L, Han ZB, Li Z, Geng J, Zhao M, Jia H, Feng J, Wei Z, Song B, Zhang J, Li J, Liu T, Wang F, Li T, Li J, Fang Y, Gao J, Han Z. Intravenous injection of allogeneic umbilical cord-derived multipotent mesenchymal stromal cells reduces the infarct area and ameliorates cardiac function in a porcine model of acute myocardial infarction. Stem Cell Res Ther 2018;9:129

28. Zhang L, Liu C, Wang H, Wu D, Su P, Wang M, Guo J, Zhao S, Dong S, Zhou W, Arakaki C, Zhang X, Zhou J. Thrombopoietin knock-in augments platelet generation from human embryonic stem cells. Stem Cell Res Ther 2018;9:194

29. Pachón-Peña G, Serena C, Ejarque M, Petriz J, Duran X, Oliva-Olivera W, Simó R, Tinahones FJ, Fernández-Veledo $\mathrm{S}$, Vendrell J. Obesity determines the immunophenotypic profile and functional characteristics of human mesenchymal stem cells from adipose tissue. Stem Cells Transl Med 2016;5:464-475

30. Serena C, Keiran N, Ceperuelo-Mallafre V, Ejarque M, Fradera R, Roche K, Nuñez-Roa C, Vendrell J, FernándezVeledo S. Obesity and type 2 diabetes alters the immune properties of human adipose derived stem cells. Stem Cells 2016;34:2559-2573

31. Kornicka K, Houston J, Marycz K. Dysfunction of mesenchymal stem cells isolated from metabolic syndrome and type 2 diabetic patients as result of oxidative stress and autophagy may limit their potential therapeutic use. Stem Cell Rev Rep 2018;14:337-345
32. Hankamolsiri W, Manochantr S, Tantrawatpan C, Tantikanlayaporn D, Tapanadechopone P, Kheolamai P. The effects of high glucose on adipogenic and osteogenic differentiation of gestational tissue-derived MSCs. Stem Cells Int 2016;2016:9674614

33. Lafosse A, Dufeys C, Beauloye C, Horman S, Dufrane D. Impact of hyperglycemia and low oxygen tension on adipose-derived stem cells compared with dermal fibroblasts and keratinocytes: importance for wound healing in type 2 diabetes. PLoS One 2016;11:e168058

34. Krishnan B, Sallam HS, Tumurbataar B, Saieva S, Baymon D, Tuvdendorj D, Micci MA, Abate N, Taglialatela G. Amelioration of hippocampal dysfunction by adipose tissue-targeted stem cell transplantation in a mouse model of type 2 diabetes. J Neurochem 2020;153:51-62

35. Qi K, Li N, Zhang Z, Melino G. Tissue regeneration: the crosstalk between mesenchymal stem cells and immune response. Cell Immunol 2018;326:86-93

36. Caplan AI. Mesenchymal stem cells: time to change the name! Stem Cells Transl Med 2017;6:1445-1451

37. Bernardo ME, Fibbe WE. Mesenchymal stromal cells: sensors and switchers of inflammation. Cell Stem Cell 2013;13: 392-402

38. Cornu M, Thorens B. GLP-1 protects $\beta$-cells against apoptosis by enhancing the activity of an IGF-2/IGF1-receptor autocrine loop. Islets 2009;1:280-282

39. Gaddy DF, Riedel MJ, Pejawar-Gaddy S, Kieffer TJ, Robbins PD. In vivo expression of HGF/NK1 and GLP-1 from dsAAV vectors enhances pancreatic B-cell proliferation and improves pathology in the $\mathrm{db} / \mathrm{db}$ mouse model of diabetes. Diabetes 2010;59:3108-3116 